\title{
On the applicability of steerable beams in LTE- Advanced networks with high user mobility
}

\author{
Christos Papathanasiou ${ }^{1 *}$, Nikos Dimitriou ${ }^{2}$ and Leandros Tassiulas ${ }^{1}$
}

\begin{abstract}
This article deals with the problem of applying scanning narrow beams to long-term evolution (LTE)-advanced networks that include in their coverage areas fast moving mobiles. The system scenario is assumed to include eNodeBs that can form multiple beams which can steer (rotate) their respective coverage areas. Additionally, the user downlink transmissions are supposed to be dynamically scheduled based on signal-to-noise plus interference ratio reports for physical resource blocks. The mobile user equipments (UEs) have multiple antenna elements which allow maximal ratio combining of the received signals. By applying coordinated transmission of steerable beams at neighboring eNodeBs, we could mitigate inter-cell interference in a multi-cell interference deployment. The coverage area and total throughput may significantly be enhanced, compared to the current LTE standard where inter-cell interference coordination technique is adopted for low-speed UEs. Furthermore, the proposed schemes involve low-complexity hardware implementation and low power consumption.
\end{abstract}

Keywords: High speed users, LTE-Advanced, Limited feedback, Multiuser diversity, Coordinated multi-point transmission, Rotated beams

\section{Introduction}

Long-term evolution (LTE), introduced in 3rd Generation Partnership Project's (3GPP) Release 8, represents a significant change to the existing $3 \mathrm{G}$ radio access networks $[1,2]$. The LTE radio access exploits the rapid variations in channel quality for using more efficiently the available radio resources in time and frequency domains. One of the main requirements of any broadband wireless system is to provide mobility, optimized for low mobility users $(0-15 \mathrm{~km} / \mathrm{h})$, supported speeds from 15 to 120 $\mathrm{km} / \mathrm{h}$ with high performance and connection for highspeed users up to $350 \mathrm{~km} / \mathrm{h}$. Inter-cell interference coordination (ICIC) is an radio resource management function, proposed in 3GPP-LTE in order to manage the allocation of physical resource blocks (PRBs) of neighboring enhanced base stations (BSs) (evolved Node B, eNodeB) and therefore reduce inter-cell interference. The coordination between neighboring eNodeBs is released via X2 Interface. Each eNodeB sends a Load Information Message to neighboring eNodeBs. In this

\footnotetext{
* Correspondence: cpapa@uth.gr

${ }^{1}$ Centre for Research \& Technology, Hellas, University of Thessaly, Thessaly, Greece

Full list of author information is available at the end of the article
}

study, we enable advanced antenna techniques, e.g., beamforming with four antenna at the transmitter and receiver side, Orthogonal frequency-division multiplexing access which is the core of the LTE downlink transmission, adaptive modulation and coding (AMC) scheme, a real-time scheduler and cooperative multipoint transmission (CoMP), an extensively discussed technique within the context of LTE-Advanced [3].

For higher mobility cases, the existence of a feedback delay from the user to the BS degrades the performance rapidly due to outdated and thus mismatched channel quality information. If the radio channel is varying fast, it can easily be understood that any kind of scheme that requires a small amount of channel feedback information will outperform a coherent beamforming scheme that requires the knowledge of the full instantaneous channel vector. It is possible that some mobiles have always better channel conditions than others. The scheduler may not schedule a particular user until its channel conditions are favorable. This causes increased scheduling latency or jitter for the user and leads to unfair resource allocation. Quality of service (QoS) classes impose stringent latency constraints. The scheduler may be forced to schedule the user even when the channel 
conditions of the user are not favorable, which leads to limited multi-user diversity gain. In opportunistic beamforming, the eNodeB simply varies the antenna gain and the mobile report their resulting time-varying signal-tonoise plus interference ratios (SINRs). A mobile will experience a relatively high SINR when the randomly varying antenna gain happens to align closely to the optimal beamforming gain of the user equipment (UE). In order to solve the problem of scheduling latency, we align as much as possible the optimal beamforming gain for all users by scanning narrow wide beams not randomly but linearly from direction $0^{\circ}$ to $\varphi_{\max }$ to cover all the area of interest. Steerable beams provide a significant range extension and considerable multipath and interference rejection. The eNodeB schedules data transmission by exploiting partial feedback on the minimum SINR per UE.

Our contribution is that only this article studies the performance of applying scanning narrow beams in the LTE-Advanced system for high-speed users with stringent QoS requirements and inter-cell interference. All previous studies in literature consider scanning beams in a single cell system with stationary users without guaranteeing QoS. The efficiency of the proposed solution is studied by simulations employing the WINNER II channel model [4]. This model includes all the main propagation effects such as path loss, shadowing, delay spread, angular spreads, spatial correlation, Doppler effects, etc. Figure 1 presents an overview of our proposed technique for evaluation. This article is structured in the following way: The current state-of-the-art and related studies are presented in the following section. The system model is

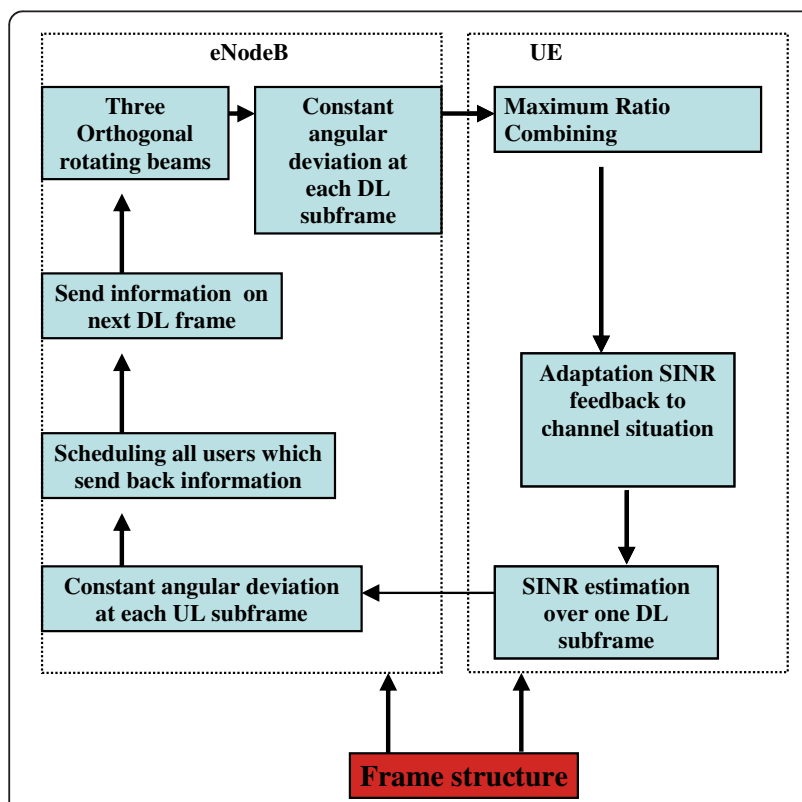

Figure 1 The proposed cross-layer (physical layer-MAC) design. described in Section "System model". The multi-cell beamforming patterns and the combining technique at the receiver are presented in Section "Beamforming pattern and combining technique". Section "SNR feedback and scheduling" introduces the feedback schemes, discusses the required frame adaptation to accommodate the rotated beams and describes the scheduling strategy. Section "Simulation results" presents the performance results that lead to the conclusions that are drawn in Section "Conclusions".

\section{Related state-of-the-art}

\section{Opportunistic beamforming}

The communications literature has significantly discussed the capacity increase that can be achieved by using scanning ams not linearly but randomly, a technique also named as opportunistic beamforming. The beams are formed without any knowledge about the position of the users. Therefore, channel quality feedback is not necessary for constructing beams (compared to the completely adaptive systems). Previous such studies have mainly been focused on stationary users. In [5], opportunistic beamforming artificially induces time fluctuations in the channel to increase the multi-user diversity. In this case, only a single user is scheduled at each time and thus one of the spatial degrees of freedom is being used. Each user $k$ feeds back the overall received signalto-noise ratio (SNR) of its own channel to the BS. The gain from multi-user diversity is related to power because the SNR of the user being scheduled is boosted accordingly. In reality, the statistics for the channels experienced by different mobiles are not similar due to different distances from the BS, shadowing effects and scattering and therefore some mobiles have always better channel conditions than others. In [6], an opportunistic space division multiple access (SDMA)-based scheduling scheme employing multiple orthogonal beams to serve multiple users simultaneously in each slot is proposed. Different low complexity combining techniques are proposed for improving the effective SINR.

\section{Receiver techniques}

In [7], the mobile station adopts minimum mean squared error receiver to exploit spatial multiplexing gain. The common channel is allocated to more than one users simultaneously. Due to separate layers, this scheme requires additional amount of feedback. In [8], the BS generates the precoding matrix $\left(V_{0}\right)$ in a controlled but pseudorandom fashion. The mobile $k$ multiplies the received signal by the left singular matrix $\left(U_{k}^{H}\right)$ of the channel matrix $H_{k}=U_{k} \Lambda_{k} V_{k}^{H}$. This method is named singular value decomposition. This scheme results in unwanted self-interference when the channel is not near its peak conditions $\left(V_{k} \neq V_{0}\right)$. In our case, the 
vector $u_{1 k}^{H}$-i.e., the first column of matrix $U_{k}^{H}$-corresponds to the strongest propagation mode and the users must not deviate from their respective peak conditions in order to achieve good SNR.

\section{1-Bit feedback information}

A single bit feedback per user mechanism is applied with one antenna element at the BS [9]. In this study, the BS selects randomly among users for data transmission. The abovementioned "1-bit algorithm" achieves the same capacity with the case of assuming full channel state information (CSI) feedback subject to a judicious choice of the threshold. However, the determination of the optimum threshold is a challenging task.

\section{Interference management}

The combination of two basic approaches, frequency/ time coordination and power coordination, is the basis for many techniques suggested in the context of broadband systems. LTE-Advanced which is an evolution of LTE introduces beamforming and CoMP technologies. CoMP, which can be seen as an extension of ICIC, joins transmission and reception across multiple cells. In the above schemes, it is difficult to find accurately the interference sources in downlink. Especially in the downlink direction, if beamforming is considering, it is necessary to determine the source and the target of interference. By coordinating the phase of the linear scanned beams, we can avoid and at the same time alleviate via the transmission of narrow beams intercell interference without the serving cell needs information from multiple cells to determine which cells mainly interfere. Scheduler does not adjust the interference PRBs and its power level as in ICIC scheduling strategy proposed in 3GPP-LTE.

\section{System model}

LTE physical (PHY) layer which transmits data and control information efficiently between an eNodeB and mobile UE is assumed. The downlink air interface is divided into a number of resource allocation elements. A frame lasts for $10 \mathrm{~ms}$. Each frame is divided into ten subframes. Each subframe has an overall length of $1 \mathrm{~ms}$ and is divided in the time domain into two slots $(0.5 \mathrm{~ms}$ duration each). The slot consists of either six or seven OFDM symbols, depending on whether the normal or extended cyclic prefix is employed. The PRB is the smallest resource allocation element, has both a time and frequency dimensions, and is assigned by the Medium Access Control (MAC) scheduler. A PRB consists of 12 consecutive subcarriers for one slot with subcarrier bandwidth $15 \mathrm{kHz}$. The number of PRBs depends on the channel bandwidth. For $20 \mathrm{MHz}$ bandwidth, the number of available PRBs is 100. A downlink multi-user MIMO-
SDMA system with $M_{\mathrm{t}}=4$ transmit antenna elements at the eNodeB and $M_{\mathrm{r}}=4$ received antenna elements at each UE is considered. Therefore, the maximum number of orthogonal beams is equal to $M_{\mathrm{t}}=4$, i.e., $M_{\mathrm{t}}=4$ users can simultaneously be scheduled in each PRB. In order to increase the total throughput of the system, we divide the hexagonal cell into three service areas $S_{i}, i \in\{1,2,3\}$. The eNodeB constructs $M_{\mathrm{t}}=3$ orthogonal rotating beams $W_{i} \in C^{N t \times 1}$ for $i=1, \ldots, M_{\mathrm{t}}$ in order to scan all the cell area. The high mobility requires limited feedback between the eNodeB and the UEs while intercell interference requires a new radio resource management function. Let $W_{i}=\left[w_{i 1}, w_{i 2}, \ldots, w_{i M t}\right]$ the orthogonal beamforming vector $M_{\mathrm{t}} \times 1$ applied at the eNodeB with $M_{\mathrm{t}}$ antenna elements. $w_{i 1}$ is the weight applied at the antenna element 1 for transmitting at service area $i$. During the $m$ th $\mathrm{PRB}$, the beamforming vector applied at the eNodeB $W_{i}(m)$ with $i=\left\{1,2,3, \ldots, M_{\mathrm{t}}\right\}$ is constant because the narrowband PRBs in frequency and time domain may experience flat fading. The transmitted signal is expressed as

$$
X_{i c}(m)=W_{i c}(m) S_{i c}(m)
$$

where $S_{i}(m)=\left[s_{i c 1}(m), s_{i 2}(m), \ldots, s_{i M \mathrm{t}}\right]^{\mathrm{T}}$ is the transmitted data vector, assuming that $E\left\{\left|S_{i}(m)\right|^{2}\right\}=1 . s_{i 1}(m)$ is the transmitted complex symbol transmitted from antenna element 1 in the $m$ th PRB for the UEs which are found in area $i$. At the beginning of each PRB, the eNodeB constructs a set of up to $M_{\mathrm{t}}=3$ orthogonal beams $W_{i}(m)$ and consequently the transmitted signal is the superposition of these beams

$$
X(m)=\sum_{i=1}^{M_{\mathrm{t}}} X_{i}(m)=\sum_{i=1}^{M_{\mathrm{t}}} W_{i}(m) S_{i}(m)
$$

The UE $k$ estimates the forward link channel matrix $H_{k}(m)$ by the special reference signals employing from LTE systems for channel estimation and designs the feedback that the eNodeB uses to adapt the transmitted signal to the channel. For notation simplicity, we drop index $m$. The downlink $M_{\mathrm{r}} \times M_{\mathrm{t}}$ channel matrix $H_{k}$ is expressed as $H_{k}=\left[H_{k, r, t}\right]$, where $H_{k, r, t}$ denotes the channel response between the receive antenna element \# $r$ of mobile $k$ and the transmit antenna element \# $t$ of eNodeB. The received signal $Y_{k} \in C^{M \mathrm{r}}$ at the $k$ th UE can be expressed as

$$
Y_{k}=H_{k} X+n_{k}+I_{k}
$$

where $n_{k}$ is the zero-mean Gaussian noise with variance $\sigma_{k}^{2}$ and $I_{k}$ is the intercell interference that the UE $k$ experiences. The co-channel interference of $M_{\mathrm{t}}-1$ interfering beams is suppressed due to orthogonality. We suppose that CSI is known at the receiver. The 
received signal is multiplied by a coefficient vector $U_{k}=$ $\left[u_{k, 1}, u_{k, 2}, \ldots, u_{k, M t}\right]^{\mathrm{T}}$ and after that it is combined. The detector calculates the vector $U_{k}$ in order to estimate the transmitted symbol

$$
\begin{aligned}
& \hat{S}_{k}=U_{k}^{H} Y_{k} \\
& \hat{S}_{k}=U_{k}^{H} h_{i k} S_{i}+U_{k}^{H}\left(n_{k}+I_{k}\right)
\end{aligned}
$$

where $h_{i k}=H_{k} W_{i}$ is the projection of beam $W_{i}$ on the channel $H_{k}$. Then, the SINR computed at the receiver $k \in S_{i}$ is

$$
\operatorname{SINR}_{i, k}=\frac{\left|U_{k}^{H} h_{i, k}\right|^{2}}{U_{k}^{2}\left(\sigma_{k}^{2}+I_{k}\right)}
$$

The achievable rate of the $k$ th UE on the $i$ th beam is given by

$$
C_{i, k}=\log _{2}\left(1+\operatorname{SINR}_{i, k}\right)
$$

According to the $\mathrm{SINR}_{i, k}$ on every PRB, different AMC schemes can be implemented.

The WINNER II channel model is a geometric-based stochastic model with separate propagation parameters and antenna elements. The large-scale parameters such as path loss and shadowing factor are generated according to the position of eNodeB and mobile UEs. The statistical channel is constructed by some distribution functions of delay and angle and also by the power delay and angular profiles extracted from channel measurements. The channel consists of a number of rays (plane waves). Each ray is delayed and weighted with a proper amplitude and phase. The waves are characterized by their Angle of Departure (AoD) and Angle of Arrival (AoA). Waves with similar AoDs, AoAs, and delays can geometrically be summed to construct a cluster. The cluster is a propagation path diffused in space, delay, and angle domains. The Clustered Delay Line (CDL) is a fixed channel model with tabulated spatio-temporal channel parameters. The CDL is a deterministic model despite the random initial phases of the rays. The Doppler spread is not explicitly defined because it results from the specific individual angular properties. Different propagation scenarios are defined. Each channel scenario dictates the spatial model parameters and is based on the WINNER measurements. These parameters are supported for center frequencies of $2-6 \mathrm{GHz}$ and bandwidths of the order of $100 \mathrm{MHz}$. The $\mathrm{C} 1$ scenario of WINNER II channel model in the metropolitan area for suburban macro-cells is used in our simulations. Path loss and shadow fading (large-scale parameters) are selected for LOS/NLOS environments. In suburban macrocells, the eNodeBs are mounted on tall building rooftops to allow wide coverage. Mobiles are often surrounded by houses and local scatterers (buildings) but occasionally they are also in open areas such as parks. For the $\mathrm{C} 1$ scenario, the LOS CDL model is given in Table 1 [4]. The statistical channel is defined by an exponential power delay profile and wrapped Gaussian (inverse Gaussian function applied) power angular profile. The AoD varies from $-33^{\circ}$ for cluster 7 to $35^{\circ}$ for clusters $8,10,12,15$.

\section{Beamforming pattern and combining technique}

Co-channel interference from neighboring cells is a fundamental limiting factor on the capacity that is also affected by channel dynamics linked to high mobility. Excessive transmit power does not improve system performance but only adds to the unnecessary co-channel interference. All the available bandwidth is allocated to each service area (e.g.. each sector) $S_{i}, i \in\{1,2,3\}$. The sector area $S_{i}$ is divided into $N_{B}$ sub-areas of equal size $\triangle S$ (a grid of $N_{B}$ beams). A narrow width beam is rotated to scan the whole area $S_{i}=360^{\circ} / 3=120^{\circ}$. A conventional beamformer is a simple beamformer with all its weights having equal magnitudes. The phases are selected to steer the antenna array in a particular direction $\phi_{0}$, known as the "look direction". Remember that beamforming is assumed to remain constant during each PRB which is the scheduling interval. The array weights for the direction of the main lobe $\phi_{0 i}$ in the area $S_{i}$ are given by

$$
w_{\phi_{0 i}}=\frac{1}{\sqrt{M_{t}}}\left[1, e^{i 2 \pi d / \lambda \sin \phi_{0 i}}, \ldots, e^{i 2 \pi\left(M_{t}-1\right) d / \lambda \sin \phi_{0 t}}\right]
$$

Table 1 LOS CDL model

\begin{tabular}{lllll}
\hline Cluster \# & Delay (ns) & Power (dB) & AoD $\left(^{\circ}\right)$ & AoA $\left(^{\circ}\right)$ \\
\hline 1 & $0|5| 10$ & $0|-25|-27$ & 0 & 0 \\
2 & 85 & -21.6 & -29 & -144 \\
3 & 135 & -26.3 & -32 & -159 \\
4 & 135 & -25.1 & -31 & 155 \\
5 & 170 & -25.4 & 31 & 156 \\
6 & 190 & -22 & 29 & -146 \\
7 & 275 & -29.2 & -33 & 168 \\
8 & $290|295| 300$ & $-24|-26|-28$ & 35 & -176 \\
9 & 290 & -23.2 & -30 & 149 \\
10 & 410 & -32.2 & 35 & -176 \\
11 & 445 & -26.5 & -32 & -159 \\
12 & 500 & -32.1 & 35 & -176 \\
13 & 620 & -28.5 & 33 & -165 \\
14 & 655 & -30.5 & 34 & -171 \\
16 & 960 & -32.6 & 35 & 177 \\
\hline
\end{tabular}


where $d$ is the distance between adjacent antenna elements in a uniform linear array (ULA) and $\lambda$ is the carrier wavelength. The angle of the steering beam $\phi_{0 i}$ increases linearly in time

$$
\phi_{0 i}(t)=\phi_{\text {init }, i}+\Delta \phi t \quad t=1,2, \ldots, N_{T}
$$

$\phi_{\text {init, } i} \in\left\{0^{\circ}, 120^{\circ}, 240^{\circ}\right\}$ is the initial value of $\phi_{0 i}$ and depends on the sector area $S_{i}$ that will be scanned, $\Delta \phi$ is the angle increment, and $N_{T}$ the period of the beamforming process.

Figure 2 illustrates the hexagonal cell deployment when three narrow beams scan the whole cell area. No intra-cell interference exists because the three beams are orthogonal. Also, we coordinate the transmission of neighborhood cells in order to combat inter-cell interference and therefore improve cell-edge performance. For example, we coordinate the initial phase $\phi_{0 i}$ of the overlapping areas of three neighborhood cells 1,2 , and 3, $\phi_{0 i}=0^{\circ}$ for cell $1, \phi_{0 i}=120^{\circ}$ for cell 2 , and $\phi_{0 i}=240^{\circ}$ for cell 3 of Figure 2. The three beams of cells 1 and 2 are rotated in the clockwise direction while the beams of cell 3 in the inverse direction. In this way, the two scanned beams which serve the cell-edge users of two neighbor cells are never overlapping which keep co-channel intercell interference $I_{k}$ at very low level. At the receiver side, it has been shown that optimum combiner (OC) and maximum ratio combiner (MRC) strategies outperform the antenna selection technique, when a limited number of users are served in opportunistic schemes with

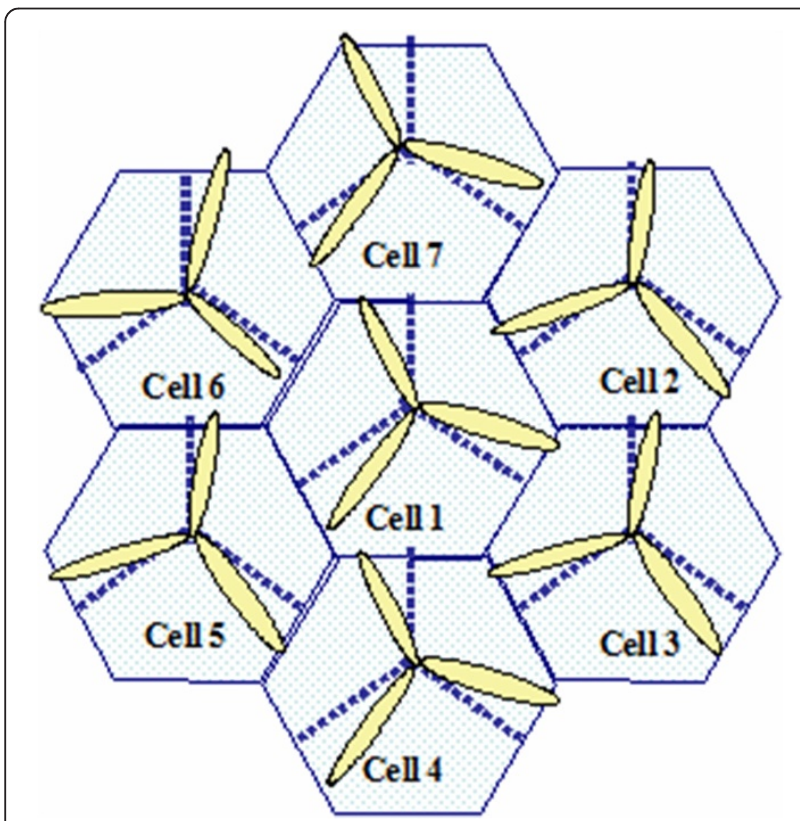

Figure 2 Cell deployment with scanning beams. multiple beams [10]. According to the OC technique, the optimum value $\Delta \mathrm{X}_{\mathrm{i}}=\mathrm{X}_{\mathrm{i}}-\widehat{\hat{X}}_{i}=\mathrm{X}_{\mathrm{i}}-\mathrm{H}_{\mathrm{k}} \widehat{S}_{i}$ of the squared estimation error is found, taking into account that the system is dominated by interference, e.g., $\sigma_{k}^{2} \rightarrow 0$. In our study, the system deployment achieves low interference level and therefore the OC method is not selected at the receiver side. With the MRC method the magnitude of the composite channel response $\left|h_{i k}\right|$ is maximized when the receiver antenna gain is aligned to the optimum value $U_{k}^{o p t}=\frac{h_{i k}}{h_{i k}}$ (the antenna is aligned to the direction of $h_{i k}$ ) and the SINR over the beam $i$ at the user $k$ is

$$
\operatorname{SINR}_{i k}^{\mathrm{MRC}}=\frac{h_{i k}^{4}}{h_{i k}^{2}\left(\sigma_{\mathrm{k}}^{2}+I_{k}\right)}
$$

In a metropolitan area, no complete line of sight (LoS) propagation exists between the eNodeB and the vehicle. The eNodeB may be mounted above rooftops to reduce the near field scattering. The LoS path is attenuated due to the appearance of diffraction at the first obstacle. The reflected signals, originated from local scatters near to the mobile, are received at a comparable power level to the attenuated direct path signal. If transmission occurs at one desired direction, multi-path power can be collected by users which are located at different directions. For example, taking the C1 scenario of WINNER II channel model used in our simulation, the channel consists of rays (plane waves) that the width of AoD is equal to $70^{\circ}$. Only this area has to be scanned by the directional beam in order to cover all of the service area of $180^{\circ}$. Therefore, the period of scanning $N_{T}$ can significantly be reduced if the users in other directions receive the multi-path power. Multi-path power is not considered as interference. Furthermore, one user can be allocated to more than one steering beams during one period of scanning (a user may receive two different beams that have different directions). Consequently, data latency could be reduced. Scanning methods use electronically steerable array antennas in order to cover the surrounding area. Each sector with $120^{\circ}$ angular width can be served by 4 to 8 narrow beams. If the signal phases in a linear array vary, the constructed beam can be steered. In an electronically steered array, programmable electronic phase shifters are used at each antenna element. By programming the required phase shift value for each element, the antenna is steered. Different approaches can be used. The Butler matrix is one of the most popular switched beam methods [11]. In the LTE market, baseband architecture using digital signal processing for implementing beamforming can be chosen. This configuration increases the flexibility but this advantage is 
offset by the high power consumption since for producing $N$ beams looking at different directions $N$ transmitters (RF/IF/baseband stages) operate in parallel. Additionally, the cost and the size of the eNodeB are increased. An additional advantage is the easy integration of our proposed method with the existing architectures in the eNodeB, since only the replacement of the RF front-end is needed. The proper input port is chosen using digitally controlled MMIC switches.

\section{SNR feedback and scheduling}

Since a large number of PRBs is used, feeding back full CSI at the transmitter is prohibitive. For a channel with frequency selective fading, the frequency dimension can also be used to schedule the users. Additionally, multiple antennas increase the number of channel state parameters. In our case, we have four transmit and four receive antennas per user connection and therefore the complex channel matrix $H_{k}$ is described by 32 parameters $(4 \times 4$ coefficients for gain and $4 \times 4$ for the delay). We consider that the feedback channel is error free and delay free. For PRB $m$, the minimum SINR per PRB at the receiver $k \in S_{i}$ of all sub-carrier $l$ (e.g., 12 sub-carriers) is fed back, $\operatorname{SINR}_{k m}^{\mathrm{min}}=\min _{l \in m} S I N R_{k l}$. Having large number of PRBs as feedback, we reduce the required feedback load, but we increase the risk of UEs feeding back unreliable information. For each PRB in the downlink direction, the eNodeB schedules all the UEs that feedback that PRB index. A possible limited feedback scheme is one where the representative rate at each PRB is computed from Equation (7) with $\mathrm{SINR}_{i k}=$ $\operatorname{SINR}_{i k m}^{\min }$ and is sent to the eNodeB from all UEs $k \in$ $\{1,2, \ldots, K\}$. If beam $i$ is assigned to UE $k$ allocating the PRB $m$, then the eNodeB will transmit at a rate $r_{i k m}$ in 12 subcarriers. A simple configuration includes only the maximum representative rate $r_{i k m}$ of all PRBs. The UE feeds back only the value of the best PRB. It is clear that a number of PRBs are not chosen by the UEs, especially when the number of PRBs is large (e.g., spectrum bandwidth is large). This results in performance degradation. In terms of total throughput, this scheme is asymptotically optimal as the number of UEs increase $(K \rightarrow \infty)$. Our scheme could adopt the mechanism presented in [9]. The eNodeB sets a threshold $\mathrm{SINR}_{\mathrm{th}}$ for all UEs and for all PRBs. The UE $k$ sends "1" to eNodeB if $\operatorname{SINR}_{i k m}^{\min } \geq S I N R_{t h}$ for each PRB $m$ when the pilot symbol is received at beam $i$. Thus, we propose and evaluate a limited feedback scheme that adapts the feedback load according to channel conditions at the least possible expense of system throughput. Figure 3 shows the MAC frame structure in FDD mode when the eNodeB in each cell is enhanced by five steerable beams in directions from 1 to 5 . The frame has a duration of $10 \mathrm{~ms}$ and is divided into
$N_{B}=10$ DL-rotated subframes and $N_{B}=10$ UL-rotated subframes. The DL-rotated subframes 0 and 5 schedule the transmission on the DL directions 1 . It is weighted by the linear-rotated vector given from (7) and (8) for $t=1$. The steering vector remains constant in direction 1 throughout the DL-rotated subframe 0 and 5. Our scheduling strategy is described as the following way: Each UE $k$ feeds back information to the eNodeB at each direction of the beam $\phi_{0 i}(t)$. The mobile UEs with high velocity send $\min _{\forall m} S I N R_{i, k, m}^{\min }$ as information for channel state, which correspond to the minimum SNR of all PRBs for one subframe. On the other hand, pedestrian or vehicular users feedback more information during one subframe, e.g., the $\min _{\forall m \in q} S I N R_{i, k, m}^{\min }$ where $q$ is a Resource Block Group (RGB) and consists of a set of consecutive in frequency and time PRBs. If $Q$ RGBs is sent as feedback information, $Q_{\text {pedestrian }}>$ $Q_{\text {vehicular }}>Q_{\text {highspeed }}$ with $Q_{\text {highspeed }}=1$. It should be noted that the UEs with minimum SINR greater than a threshold are considered only candidates, since scheduler does not guarantee that all UEs are being allocated PRBs. Since statistical information is sent back to eNo$\mathrm{deB}$, we are interested in the number of PRBs that may be allocated to a UE in every RGB and do not the best PRB. To obtain highest possible spectral efficiency maximum throughput by adding fairness to UEs under bad average channel conditions, scheduler can operate in to the following mode: UEs are sorted according to min $\forall m \in q S I N R_{i, k, m}^{\min }$. Only one PRB is scheduled at each UE according to minimum SINR priority metric until the UE throughput required is satisfied based on the number of PRBs for a transmission time interval equal to 1 ms. After PRBs allocation, link adaptation chooses different modulation and coding (MCS) schemes based on minimum SINR reports in every RGB to maximize spectral efficiency.

In LTE, the UE reports to the eNodeB the highest MCS that ensures Block Error Rate $\leq 10^{-3}$ instead to SINR. The list of modulation schemes and code rates which can be signaled by means of a CSI value is shown in Table 2 [12]. The CSI values are reported by the UE into two modes: a periodic and an aperiodic one. The UE reports a CSI for the total bandwidth (this type is named wideband feedback) and for each RGB. The CSI information for each RGB is encoded differentially (with respect to the wideband) using 2 bits. For each RGB, possible values are $\{\leq-1,0,+1, \geq 2\}$. For the wideband periodic CSI reporting, the period can be configured for FDD to $\{2,5,10,16,20,32,40,64,80,160\}$ ms. The physical uplink control channel is used for periodic CSI reporting mode while the physical uplink shared channel is used for aperiodic reporting of CSI where the eNodeB instructs the UE to send an individual CSI report into a resource scheduled for uplink data transmission. 


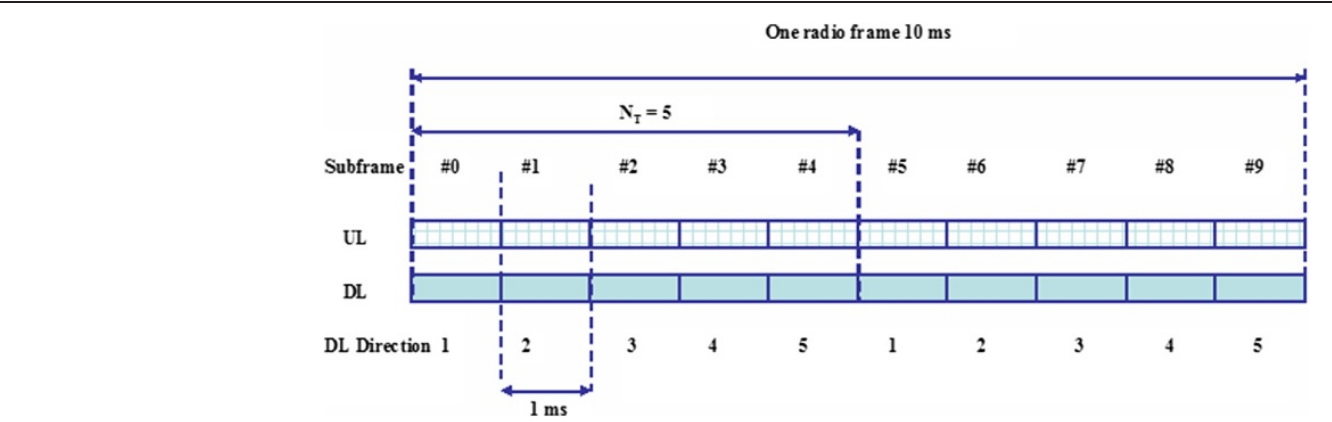

Figure 3 FDD frame structure with steerable beams.

\section{Simulation results}

This study focuses on the performance for the downlink direction. We used the parameters for the LTEAdvanced system channel as Table 3 . The speed is equal to $110 \mathrm{~km} / \mathrm{h}$ for all mobiles (see Table 3 ) and the direction of the motion is $U(-180,180)^{\circ}$ with respect to broadside, where $U$ denotes uniform pdf. Different scenarios are simulated in the following analysis. An overview of the scenarios is given in Table 4. Simulation scenario I defines a cell with one eNodeB and 30 mobile UEs randomly positioned on each sectors, e.g., 90 users on the cell. ULAs with half wavelength spacing are used at both ends. Simulation scenarios II-IV correspond to specific cases with one or three users in order to evaluate SINR versus azimuth and coverage area. As a first step, these specific scenarios are evaluated and in the end results for simulation with more users (scenario I) are given. Let us first have a look at the special case where one UE moves across a circular path with radius $R=1 \mathrm{~km}$ (scenario $\mathrm{II}$ ). We diagonalize the channel matrix $H_{k}\left(H_{k}=U_{k} \Lambda_{k} V_{k}^{H}\right)$ for the different locations with azimuth $\phi=270^{\circ}, 310^{\circ}, 0^{\circ}, 50^{\circ}, 90^{\circ}$. Figure 4 illustrates the optimum transmit eigenvector which corresponds to the strongest propagation mode and is obtained from the first column of $V_{k}$. We observe that the strongest eigenvector $V_{1}$ has azimuth range between $\phi=340^{\circ}$ and $\phi=20^{\circ}$. These results are explained from the CDL channel model in Table 1 , where the AoD of 16 clusters vary from $-33^{\circ}$ to $35^{\circ}$. In order to cover one sector area from $\phi=300^{\circ}$ to $\phi=60^{\circ}$, we form steering beams from direction $\phi=340^{\circ}$ to $\phi=20^{\circ}$. The region of interest (covering the AoD of the different scatterers) is scanned by three beams with steering directions as is shown in Figure 5.

In order to obtain also deep fades in the simulated channel profiles, we set in the WINNER II channel

Table 2 CSI table

\begin{tabular}{lll}
\hline CSI index & Modulation & Approximate code rate \\
\hline 0 & No transmission & - \\
1 & QPSK & 0.076 \\
2 & QPSK & 0.12 \\
3 & QPSK & 0.19 \\
4 & QPSK & 0.3 \\
5 & QPSK & 0.44 \\
6 & QPSK & 0.59 \\
7 & 16QAM & 0.37 \\
8 & 16QAM & 0.48 \\
9 & 16QAM & 0.6 \\
10 & 64QAM & 0.45 \\
11 & 64QAM & 0.55 \\
12 & 64QAM & 0.65 \\
13 & 64QAM & 0.75 \\
14 & 64QAM & 0.85 \\
15 & 64QAM & 0.93 \\
\hline
\end{tabular}

Table 3 System model parameters

\begin{tabular}{ll}
\hline Parameter & Value \\
\hline Cell radius (m) & 3300 \\
Frequency band (GHz) & 5.25 \\
$\begin{array}{l}\text { Number of eNodeB array } \\
\text { antenna elements }\end{array}$ & 4 \\
$\begin{array}{l}\text { Number of UE array } \\
\text { antenna elements }\end{array}$ & 4 \\
Mobile velocity (km/h) & 110 \\
Channel bandwidth (MHz) & 10 \\
Number of scanning beams & 3 \\
Cellular layout & Hexagonal grid, seven sites, three \\
Number of data subcarriers & sectors per site, wrap-around \\
eNodeB transmit power (mW) & 600 \\
Channel profile & 250 \\
UE distribution & WINNER II C1 metropolitan \\
Traffic model & Uniform, random positioning \\
\hline
\end{tabular}


Table 4 Overview of simulation scenarios

\begin{tabular}{ll}
\hline Scenario & Description \\
II & 30 users randomly positioned in each of three sectors per cell \\
III & One user on a circular trajectory with radius $1 \mathrm{~km}$ \\
IV & One user moves along the horizontal axis (azimuth always $0^{\circ}$ ) \\
& $\begin{array}{l}\text { Two users move along one axis with azimuth always } 60^{\circ} \text { and } \\
\text { the other with azimuth always } 300^{\circ} \text { and another user } \\
\text { along the horizontal axis (azimuth } 0^{\circ} \text { ) }\end{array}$ \\
\hline
\end{tabular}

model the parameter 'sample Density' equal to 64, which means that 128 channel samples per wavelength are taken. The total number of time samples is 1,000 . The output of the channel is taken in the time domain. The frequency domain output is taken by applying the FFT algorithm. The maximum frequency depends on the number of samples. We obtain the bandwidth of 10 $\mathrm{MHz}$ by applying zero-order hold interpolation with oversampling factor 198. We do not take double-sided spectrum because it is symmetric to the central frequency of operation $f_{\mathrm{c}}=5.25 \mathrm{GHz}$. Therefore, we double the power of each frequency component. After oversampling, we have 990,000 points at the frequency axis.

The average SNR versus azimuth angle for scenario II is shown in Figure 6. The SNR at each UE $k$ is calculated. It can be observed that anywhere on the circular trajectory of scenario II, an SNR between 12 and $15 \mathrm{~dB}$ is measured. We remark that the service area (from $\phi=300^{\circ}$ to $\phi=60^{\circ}$ ) can be covered by three beams with directions $\phi=0^{\circ}, 20^{\circ}$ and $-20^{\circ}$ with $3 \mathrm{~dB}$ variation approximately in SNR when the mobile is located $3 \mathrm{~km}$ (not $1 \mathrm{~km}$ as in scenario II) away from the eNodeB. The reason is that in an NLOS environment, due to the reflections and diffusion of emitted energy in different directions the azimuth angle of the first eigen beam has

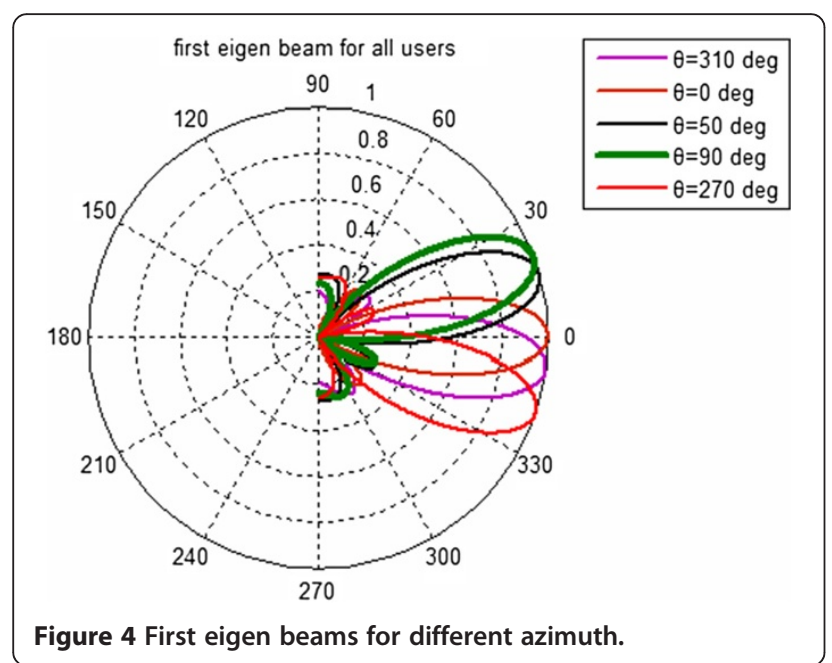

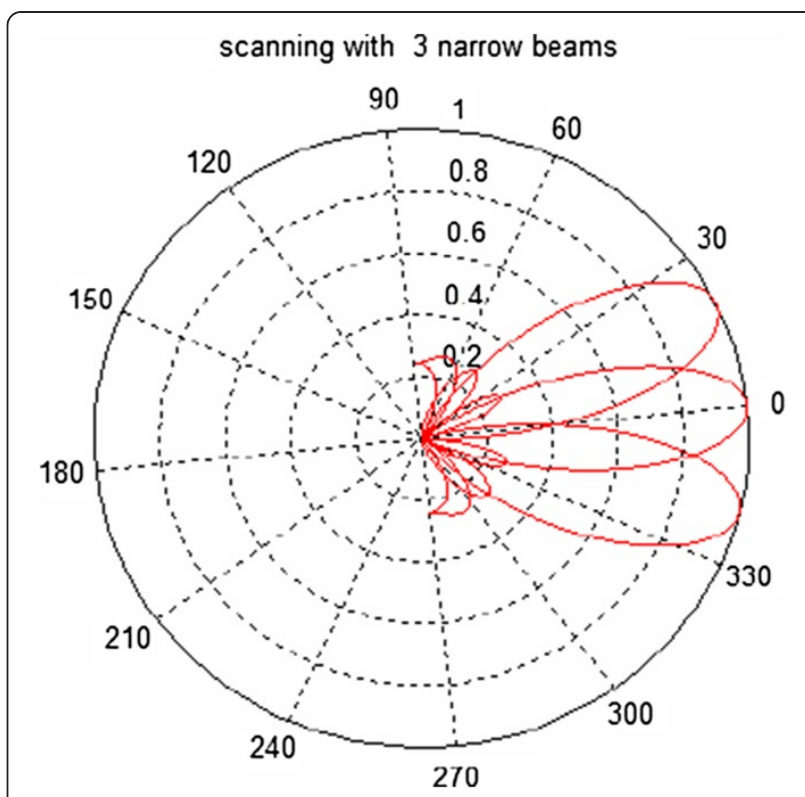

Figure 5 Scanning with three narrow beams.

a range of $40^{\circ}$ as we can see from Figure 4. Figure 7 shows the average physical layer data rate as a function of the distance for two different scenarios and the $0^{\circ}$ omni-directional antenna at the eNodeB (single input multiple output-SIMO) case. In the worst case scenario III, one user moves along the horizontal axis. Scenario IV with three users is the best case. Our scheme for scenario IV achieves a $55 \%$ improvement of range (minimum data rate $1 \mathrm{Mbits} / \mathrm{s}$ ) over SIMO $1 \times 4$ because narrow beams concentrate the power in one path which corresponds to strongest eigen mode. To identify the gains from scanning and beamforming in term of cell

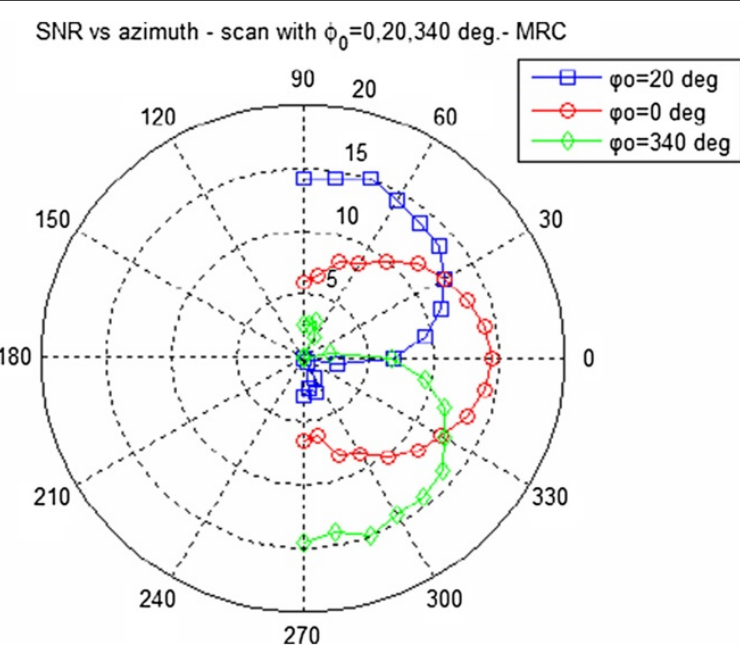

Figure 6 SNR versus azimuth angles. 


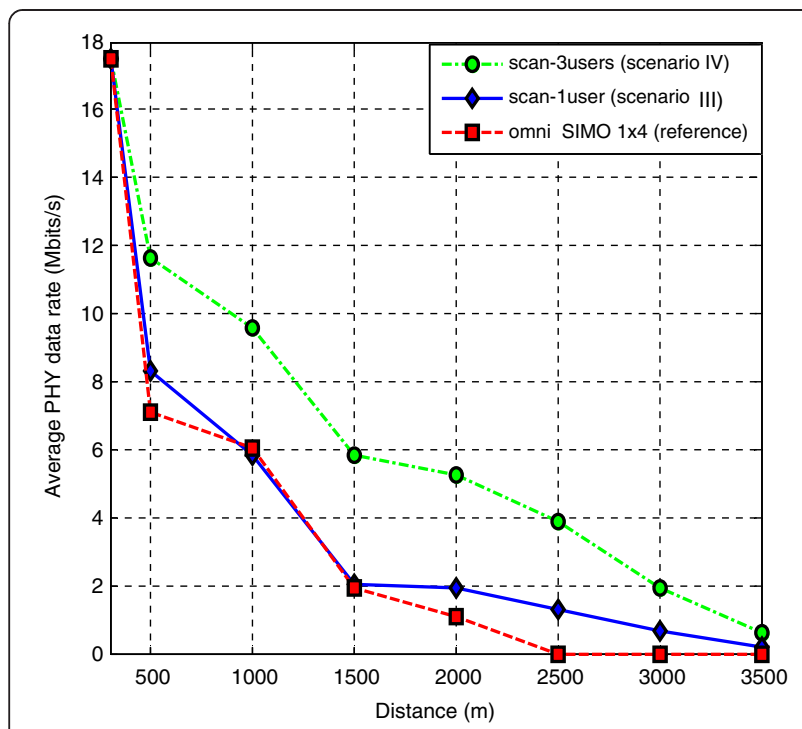

Figure 7 PHY data rate versus distance.

throughput and fairness, in the following, scenario I is used for simulation. Figure 8 shows total cell throughput for different amounts of feedback. The system capacity can significantly be enhanced using feedback compared to the system without feedback information where three $120^{\circ}$ directional antenna with frequency reuse factor equal to 3, 1/2 QPSK MCS scheme, and randomly allocation of PRBs strategy are established. These throughput numbers do not take into account the throughput loss due to signaling, e.g., throughput is measured at PHY and not at the MAC layer. The results are explained from the fact that our strategy with steerable beams mitigates inter-cell and intra-cell interference, increases the received SINR and exploits the partial CSI efficiently. In Figure 9, simulations have been performed with three and five scanning beams. The performance is similar in the two cases because the width of three steerable beams is enough to cover the area of interest. In Figures 8 and 9, total throughput decreases proportionally with the number of UEs (cell load). This fact is explained from our transmission scheduling strategy. In order to guarantee QoS, we schedule all UEs which send back information and not only the UEs with best channel conditions. When the number of cell UEs increases, the possibility some mobiles have not very good SINR increases which reduces the total throughput. We studied the effect of feedback delay by calculating the throughput per user for six users in scenario I. Figure 10 plots the degradation of throughput per user in case of ideal feedback delay, $(\delta=0 \mathrm{~ms})$, half frame $(\delta=5 \mathrm{~ms})$, and one frame $(\delta=10 \mathrm{~ms})$ delayed feedback information. In the worst case, a 10-ms delay causes a throughput loss of $10 \%$ per user while a 5-ms delay does not significantly change the user rate. Finally, Figure 11 shows the CDF of user throughput for our proposed scheme and for the SIMO $1 \times 4$ scheme. It can be seen that the fairness is improved among the users for rotated beams compared to the omni-directional transmission scheme as expected. In the omni-directional scheme, $30 \%$ of the users cannot transmit while in the first scheme only $8 \%$

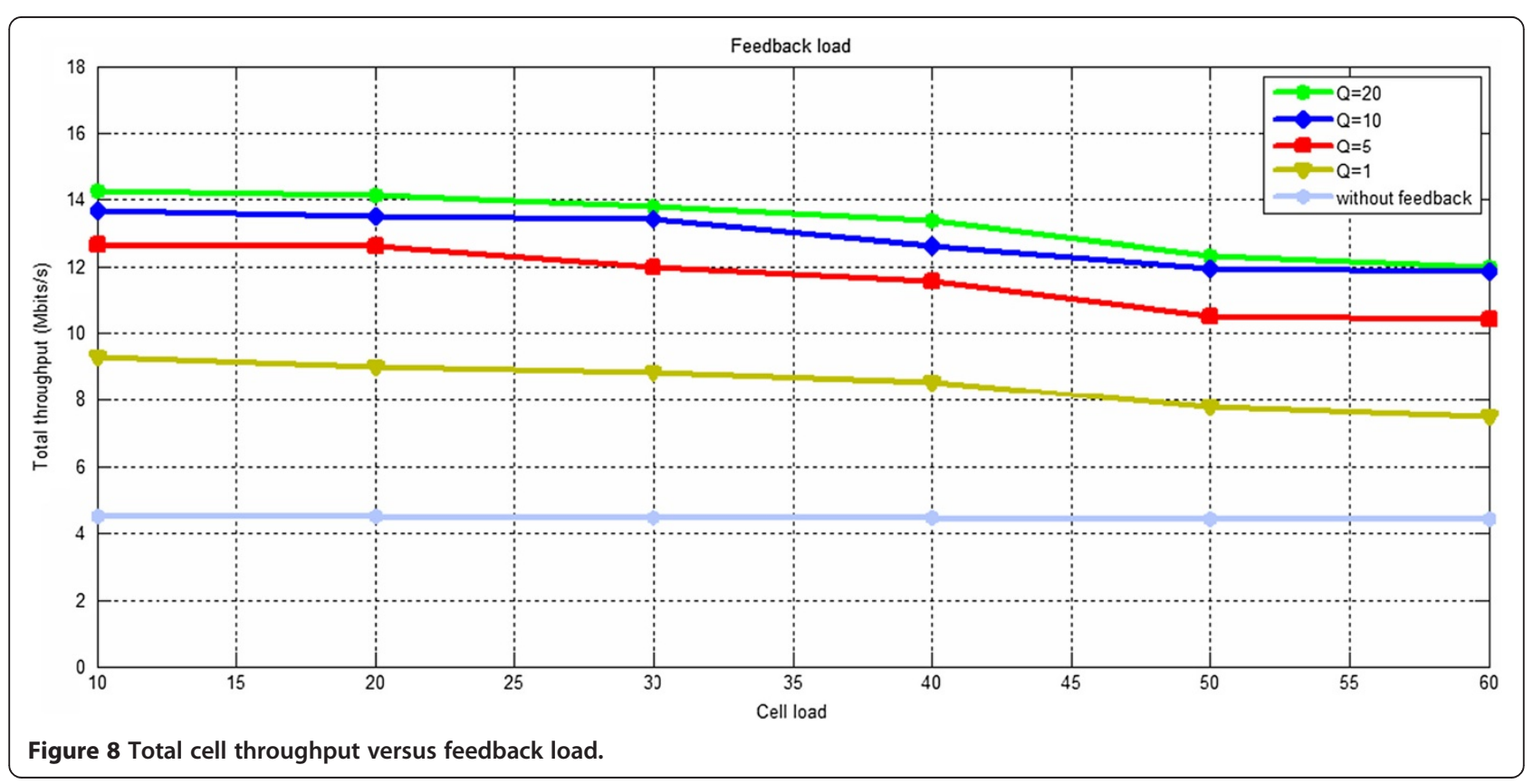




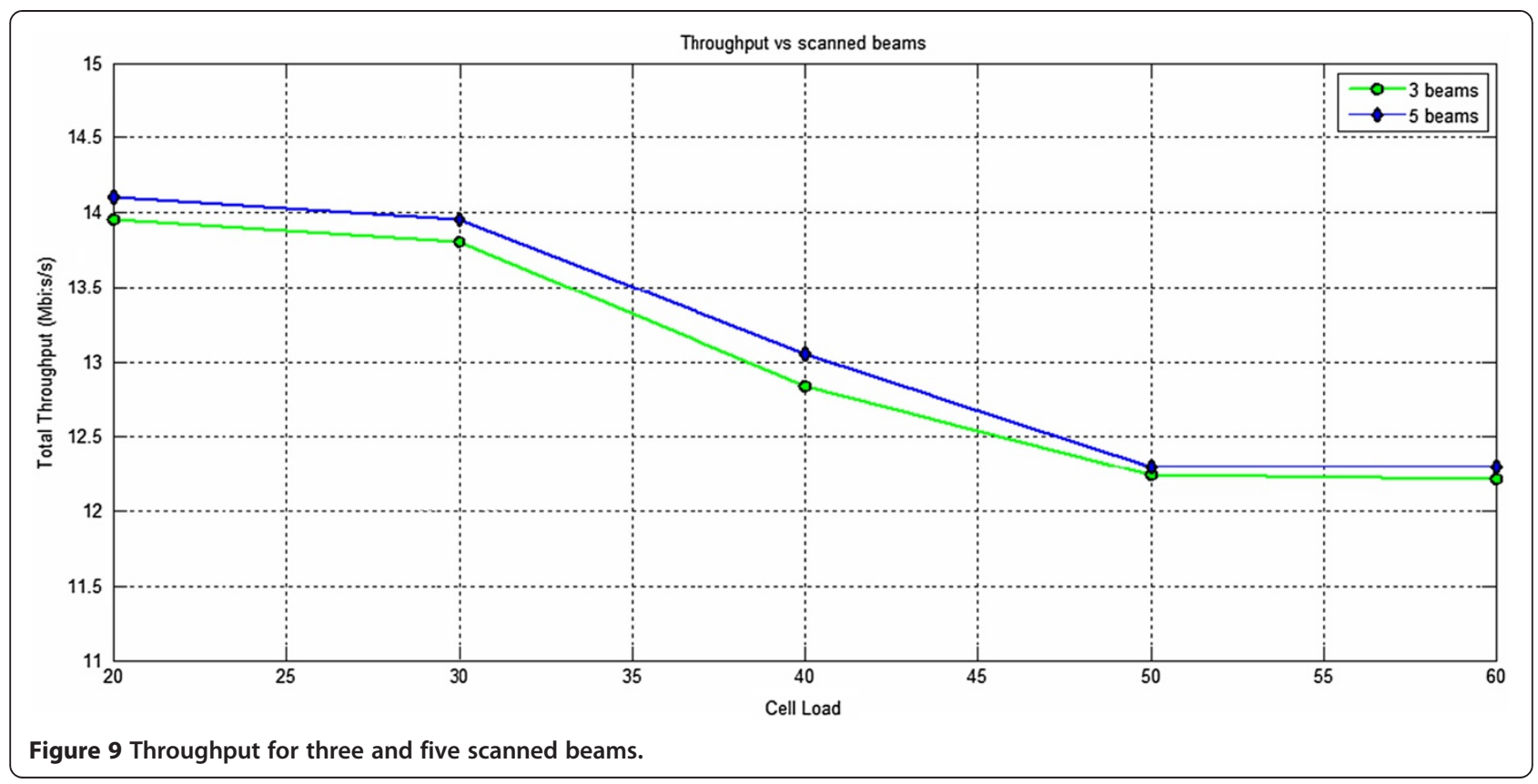

of the users do not establish connection. For higher user throughput, the CDF shift is smaller.

\section{Conclusions}

In this study, we have investigated an LTE-Advanced downlink transmission strategy that refers to the beamsteering method, feedback design, scheduling, and CoMP technology to improve the communication between high-speed vehicles and the eNodeB and combat inter-cell interference. These four mechanisms are closely coupled. It was shown that the proposed scheme significantly enhances the coverage area, the overall system throughput, and fairness among the mobile UEs. The performance of the system depends on the CSI that is provided through feedback. Additionally, we studied a CoMP technique that provides a considerable interference rejection, supporting higher users' throughput. The simulation results show that the total throughput does not increase proportionally to the number of resource block groups $(Q)$ sending as feedback information and is approximately constant if the number of fixed beams that are scanned in each sector is greater than a

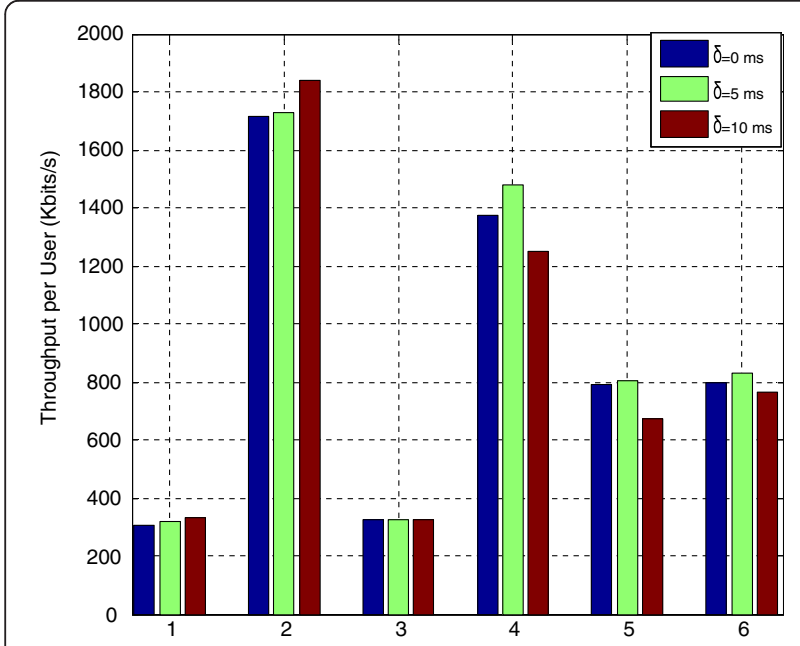

Figure 10 Difference between estimated throughput per user and instantaneous throughput user at time $t-\delta$.

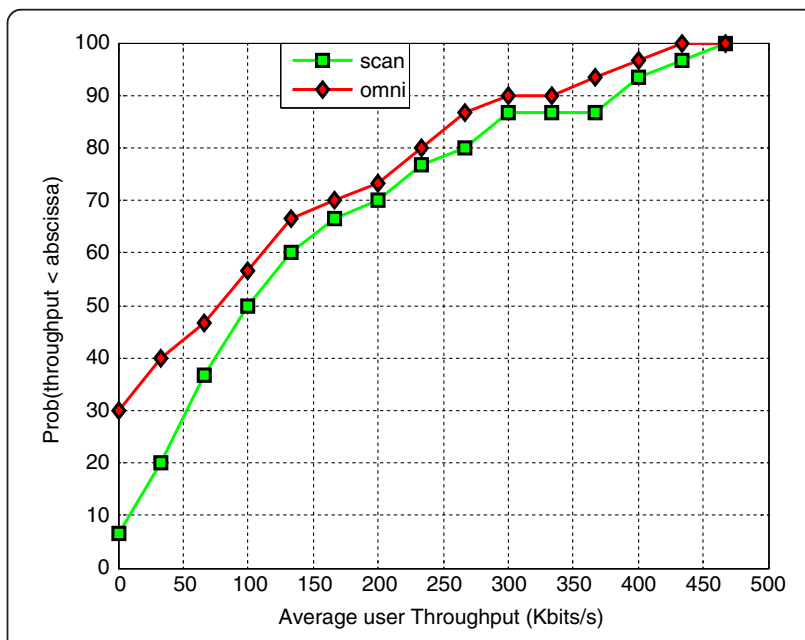

Figure 11 User throughput distribution. 
minimum value. Finally, the system throughput is reduced as function of the cell load.

In the future, a study of rotated beams in a radio-overfiber network will be done in order to provide real broadband access to very fast users like train passengers. We will also continue study towards introducing novel CoMP combining with steerable beams.

\section{Competing interests}

The authors declare that they have no competing interests.

\section{Acknowledgments}

This study was supported by the Greek Government under NSRF 2007-2013 COOPERATION WiSe-PON 09SYN-71-11555 and by European Commission under STREP CONECT FP7-257616.

\section{Author details}

${ }^{1}$ Centre for Research \& Technology, Hellas, University of Thessaly, Thessaly, Greece. ${ }^{2}$ Institute of Accelerating Systems \& Applications, National \& Kapodistrian University of Athens, Athens, Greece.

Received: 29 March 2012 Accepted: 21 June 2012

Published: 27 July 2012

\section{References}

1. 3GPP TS 36.300, Evolved Universal Terrestrial Radio Access(E-UTRA) and Evolved Universal Terrestrial Radio Access Network (E-UTRAN) Overall description, 2009.

2. 3GPP TS 36.300 V10.1.0, Technical specification, "Evolved Universal Terrestial Radio Access (E-UTRA) and Evolved Universal Terrestial Radio Access Network (E-UTRAN); Overall Description", stage 2 (Release 10) (2010-09).

3. A. Ghosh, R. Ratasuk, B. Mondal, N. Mangalvedhe, T. Thomas, LTE-advanced: next-generation wireless broadband technology. IEEE Wirel Commun 17, 10-22 (2010).

4. P. Kyosti, J. Meinila, L. Hentila, X. Zhao, T. Jamsa, C. Schneider, M. Narandžić, M. Milojević, A. Hong, J. Ylitalo, V.-M. Holappa, M. Alatossava, R. Bultitude, Y. de Jong, T. Rautiainen, IST-4-027756 WINNER II Deliverable 1.1.2. v.1.2, WINNER II Channel Models, in Part I Channel Models and Part II Channel Measurement and Analysis Results, 2007. Technical report, IST WINNERII.

5. P. Viswanath, D. Tse, R. Larroia, Opportunistic beamforming using dump antennas. IEEE Trans. Inf. Theory 48(6), 1277-1294 (2002).

6. M.-O. Pun, V. Koivunen, H. Poor, Opportunistic scheduling and beamforming for MIMO-SDMA downlink systems with Linear Combining, in PIMRC'07 Athens, Greece, pp. 1-6.

7. E.Y. Kim, J. Chun, Random beamforming in MIMO systems exploiting efficient multiuser diversity, in VTC Stockholm, Sweden, vol. 1, 2005, pp. 202-205.

8. E.Y. Kim, J. Chun, A random beamforming technique in MIMO systems exploiting multiuser diversity. IEEE J. Sel. Area Commun. 21(5), 848-855 (2003).

9. S. Sanayei, A. Nosratinia, Exploiting multiuser diversity with only 1-bit feedback, in WCNC, IEEE March 2005, vol. 2, 2005, pp. 978-983. New Orleans, LA.

10. R. Bosisio, U. Spagnolini, On the sum-rate of opportunistic beamforming schemes with multiple antennas at the receiver, in ICC 07 Glasgow, Scotland, pp. 1048-1053.

11. J. Butler, R. Lowe, Beam forming matrix simplifies design of electronically scanned antennas. Electron. Des. 9(7), 170-173 (1961).

12. S. Sesia, I. Toufik, M. Baker, LTE - The UMTS Long Term Evolution, From Theory to Practice, Chapter 10 (Wiley, New York, 2009).

doi:10.1186/1687-1499-2012-234

Cite this article as: Papathanasiou et al: On the applicability of steerable beams in LTE-Advanced networks with high user mobility. EURASIP Journal on Wireless Communications and Networking 2012 2012:234.

\section{Submit your manuscript to a SpringerOpen ${ }^{\odot}$ journal and benefit from:}

- Convenient online submission

- Rigorous peer review

- Immediate publication on acceptance

- Open access: articles freely available online

- High visibility within the field

- Retaining the copyright to your article

Submit your next manuscript at $>$ springeropen.com 